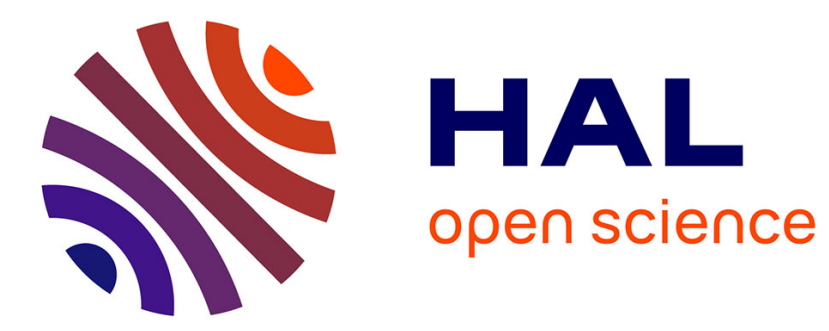

\title{
The drainage of foamy granular suspensions
}

Benjamin Haffner, Yacine Khidas, Olivier Pitois

\section{To cite this version:}

Benjamin Haffner, Yacine Khidas, Olivier Pitois. The drainage of foamy granular suspensions. Journal of Colloid and Interface Science, 2015, 458, pp.200-208. 10.1016/j.jcis.2015.07.051 . hal-01188866

\section{HAL Id: hal-01188866 https://hal.science/hal-01188866}

Submitted on 31 Aug 2015

HAL is a multi-disciplinary open access archive for the deposit and dissemination of scientific research documents, whether they are published or not. The documents may come from teaching and research institutions in France or abroad, or from public or private research centers.
L'archive ouverte pluridisciplinaire HAL, est destinée au dépôt et à la diffusion de documents scientifiques de niveau recherche, publiés ou non, émanant des établissements d'enseignement et de recherche français ou étrangers, des laboratoires publics ou privés. 


\title{
The drainage of foamy granular suspensions
}

\author{
B. Haffner ${ }^{1}$, Y. Khidas ${ }^{2}$ and O. Pitois ${ }^{1}$ \\ ${ }^{1}$ Université Paris Est, Laboratoire Navier, UMR 8205 CNRS - École des Ponts ParisTech - IFSTTAR \\ cité Descartes, 2 allée Kepler, 77420 Champs-sur-Marne, France. \\ benjamin.haffner@ifsttar.fr; olivier.pitois@ifsttar.fr \\ ${ }^{2}$ Université Paris Est, Laboratoire Navier, UMR 8205 CNRS - École des Ponts ParisTech - IFSTTAR \\ 5 bd Descartes, 77454 Marne-la-Vallée Cedex 2, France. \\ yacine.khidas@ifsttar.fr
}

Corresponding author:

Olivier Pitois

Université Paris Est, Laboratoire Navier, UMR 8205 CNRS - École des Ponts ParisTech - IFSTTAR cité Descartes, 2 allée Kepler, 77420 Champs-sur-Marne, France tel: 33181668451

email: olivier.pitois@ifsttar.fr 


\begin{abstract}
:
Foam-based materials are promising micro-structured materials with interesting thermal and acoustical properties. The control of the material morphology requires counteracting all the destabilizing mechanisms during their production, starting with the drainage process, which remains to be understood in the case of the complex fluids that are commonly used to be foamed. Here we perform measurements for the drainage velocity of aqueous foams made with granular suspensions of hydrophilic monodisperse particles and we show that the effect of particles can be accounted by two parameters: the volume fraction of particles in the suspension $\left(\varphi_{p}\right)$ and the confinement parameter $(\lambda)$, that compares the particle size to the size of passage through constrictions in the foam network. We report data over wide ranges for those two parameters and we identify all the regimes and transitions occurring in the $\varphi_{p}-\lambda$ diagram. In particular, we highlight a transition which refers to the included / excluded configuration of the particles with respect to the foam network, and makes the drainage velocity evolve from its minimal value (fully included particles) to its maximal one (fully excluded particles). We also determine the conditions $\left(\varphi_{p}, \lambda\right)$ leading to the arrest of the drainage process.
\end{abstract}

Keywords : foam; suspension; granular; particle; jamming; aerated materials 


\section{Symbols}

$\varphi_{p}:$ volume fraction of particles in the interstitial phase of the foam

$\varphi_{p}^{\text {pack }}$ : volume fraction of packed spheres

$D_{b}$ : bubble size

$\phi$ : gas volume fraction

$d_{p}:$ particle diameter

$d_{c}$ : diameter of passage through constrictions in the foam network

$\lambda=d_{p} / d_{c}:$ confinement parameter

$r$ : characteristic size of a foam node

$V:$ drainage velocity

$\tilde{V}=V\left(\varphi_{p}\right) / V(0):$ reduced drainage velocity

$N$ : number of particles per foam node

$\mu_{0}, \mu$ : shear viscosity of the suspending liquid, of the interstitial suspension

$K$ : foam permeability

$\tilde{k}_{n}=K / r^{2}$ : permeability coefficient of a foam node 


\section{Introduction}

Foaming is widely encountered in industrial processes: gas is mixed to many materials in order to improve their thermal or acoustical performance or simply to make them lighter and to save raw materials. In the current climate of sustainable development, the production of foam-based materials is destined to expand. The matrix of those foamy materials is often composed of a complex fluid, such as a suspension for example. Typical examples for such mixtures can be found in the production of materials for the building industry [1], of ceramic foams [2], or in food [3] and cosmetic industries. Note also that the mining industry extensively resorts to mixtures of foam and particles through the flotation process that is widely used to separate ores [4].

The homogeneity of foamy materials can be drastically affected by the drainage of the interstitial phase (the continuous phase between the gas bubbles) and the simultaneous rising of the bubbles, resulting in the degradation of their quality and their functional properties. Note also that the drainage of the liquid phase - and the resulting increase of the gas volume fraction - promotes other detrimental aging processes, such as ripening and coalescence $[5,6]$. In order to control foambased materials it is crucial to understand and to counteract as much as possible the drainage process. During the last two decades, most of the work realized in the field of foam drainage has concerned aqueous foams, i.e. dispersions of densely packed gas bubbles in a Newtonian liquid [57]. In fact, only a few recent studies have tackled the issue of foam drainage with complex fluids, such as clays [8,9], coal fly ashes [10], colloidal suspensions [11-14], emulsions [15-17]. These studies have highlighted finite size effects and particle trapping phenomena (clogging) occurring at the scale of the foam network.

Very recently, some of these effects have been rationalized thanks to experiments with model systems, and the so-called confinement parameter $\lambda$ has been identified as a control parameter. $\lambda$ compares the size of particles contained in the interstitial phase to the size of passage through the constrictions in the interstitial network: $\lambda=d_{p} / d_{c}$ (see Fig. 1). Two mechanisms for trapping of particles in aqueous foams have been understood: (i) the collective trapping - jamming - of the suspension for $\lambda<1$ and for particle volume fractions above of a critical value that depends on $\lambda$ [18], and (ii) the individual capture of the particles by the foam constrictions for larger $\lambda$ values [1921].

These two mechanisms give some insight into the drainage of foams in the presence of suspended particulate matter. However, the complete understanding of drainage laws requires more experimental work with such model systems. In this paper, we perform new measurements for the 
drainage velocity of aqueous foams in the presence of spherical particles. Thanks to an improved sample's generation method we obtain a new set of data for large $\lambda$ values - up to $20-$ and we complete our previous data obtained for $\lambda<2$. This allows for a global physical picture to be proposed for the drainage of foamy suspensions.

\section{Materials and methods}

Samples are prepared from precursor liquid foams which are subsequently mixed with granular suspensions, as described in a previous work $[18,21]$.

\section{Materials}

The foaming solution contains $10 \mathrm{~g} / \mathrm{L}$ of trimethyl(tetradecyl)azanium bromide (TTAB) in distilled water with $20 \% \mathrm{w} / \mathrm{w}$ glycerol. With such a proportion of glycerol the density of the solution is 1050 $\mathrm{kg} / \mathrm{m}^{3}$ and matches with that of polystyrene particles used in the study. The surface tension of the liquid/gas interface is $38 \mathrm{mN} / \mathrm{m}$ and shear viscosity of the bulk is $\mu_{0} \simeq 1.7 \mathrm{mPa}$.s. The suspension is prepared at a given particle volume fraction $\left(\varphi_{2}\right)$ by mixing the foaming solution and polystyrene spherical beads (Microbeads ${ }^{\circledR}$ ). The beads are quite monodisperse: $\Delta d_{p} / d_{p} \approx 5 \%$ and we have used the following diameters: $d_{p}=6,20,30,40,80,140,250$ and $500 \mu \mathrm{m}$. In the foaming solution, those particles behave as fully hydrophilic particles and they do not adsorb at bubble interfaces.

\section{Generation of the precursor foams (schema 1 in Fig. 2)}

Bubbles are generated in a T-junction with two entries (nitrogen and foaming solution) and one exit (bubbly solution). The bubble diameter was varied in the range $D_{b} \simeq 150-1000 \mu \mathrm{m}$ by tuning the flow rates of gas and liquid. The bubbles are continuously produced and released at the bottom of a column which is partially filled with the foaming solution. This results in the formation of foam in the column. During the production, the foam is imbibed with the same foaming solution in order to obtain stationary drainage conditions with a constant value of the gas fraction $\left(\phi_{1}\right)$ throughout the foam column $[5,6,22]$.

\section{Mixing of the precursor foams with the granular suspensions (schema 2 in Fig. 2)}

Once the column is filled, the foam is flushed towards a mixing device where the granular suspension is introduced. We have checked that the mixing device does not break bubbles. The parameters of the resulting foamy suspension are controlled by the relative flow rates of the precursor foam $\left(q_{1}\right)$ and the suspension $\left(q_{2}\right)$. The typical value for $q_{1}$ is $10 \mathrm{~mL} / \mathrm{min}$. The final gas fraction $\phi=$ 
$q_{1} \phi_{1} /\left(q_{1}+q_{2}\right)$ was varied in the range $0.85-0.95$, but most data were obtained for $\phi=0.90$. We consider the volume fraction of particles in the interstitial phase: $\varphi_{p}=q_{2} \varphi_{2} /\left[q_{1}\left(1-\phi_{1}\right)+q_{2}\right]$. The outlet of the mixing device is connected to a cylindrical tube (inner diameter $26 \mathrm{~mm}$ ) in which the produced foamy suspension is continuously introduced. It is equipped with a piston which rate for withdrawing motion compensates exactly the volume flow rate of the injected foamy suspension. Moreover, the tube is rotated $(0.3 \mathrm{~Hz})$ along the horizontal axis in order to compensate the effects of gravity during the filling step. We stop this step once the volume of produced particulate foam equals $\Omega_{\text {tot }} \simeq 60 \mathrm{~mL}$, which corresponds to a foam length approximately equal to $11.5 \mathrm{~cm}$.

\section{Study of drainage (schema 3 in Fig. 2)}

Then the foam tube is turned to the vertical and we start to measure the drainage kinetics of the sample. We follow the evolution of the height $h(t)$ locating the transition between the foam and the drained suspension at the bottom of the column (see Fig. 3). Note that the pictures such than those presented in Fig. 3 were used to check for the absence of coalescence during the measurements. As already pointed out $[18,21]$ the drainage behavior of foamy suspensions exhibits a linear regime, characterized by a well-defined and constant drainage velocity, provided that $t \lesssim \tau$, where $\tau$ is the half drainage time. During this regime, the volume of liquid/suspension drained out of the foam has flowed through foam areas that have not yet been reached by the drainage front, i.e. areas where the gas fraction has remained equal to the initial value $\phi$. Because the linear regime accounts for drainage properties of foam characterized by a constant gas fraction $\phi$, we measure the drainage velocity $V$ from the slope of this linear evolution, $V=d h / d t$. Note that of all drainage curves were found to exhibit the linear regime, as shown in Fig. 3. In order to characterize the effect of particles on drainage, we normalize the measured drainage velocity by the one measured without particle, i.e. $\tilde{V} \equiv \mathrm{V}\left(\varphi_{p}\right) / \mathrm{V}(0)$. Note that because of uncertainties related to the measurement of $h(t)$ for $h \simeq 0$, linear fits are not applied to the early stage of the linear regime. The maximum relative error on the reduced drainage velocity is estimated to be close to $15 \%$.

\section{Two-step method (Fig. 2)}

The mixing method reaches practical limits for high concentrations of large particles, i.e. $\varphi_{p} \gtrsim 0.35$ and $\lambda \gtrsim 2$, so that we have developed an alternative method, the so-called two-step method, for preparing samples with such parameters. The first step is identical to the one described above (schemas 1-2-3 in Fig. 2) except that gas and particle fractions, $\phi_{i}$ and $\varphi_{p_{i}}$, are lower than their final target values $\phi$ and $\varphi_{p}$. As this method applies to rather high $\lambda$, the particles remain trapped in the foam during the drainage of the suspending liquid. After the drainage step, the most part of the 
drained liquid is withdrawn from the bottom of the foam sample and, in order to homogenize the sample, the column is returned to the horizontal and the rotation motion is restored. Then, a given volume of that withdrawn liquid is re-injected at a small flow rate into the rotating foam column. The difference between withdrawn and re-injected liquid volumes is $\Delta \Omega_{l}$. Note that if the drained liquid was not almost totally removed from the column and then partially re-injected, the particles would be displaced by the strong imbibition front occurring when the column was returned to the horizontal position. Our procedure allows for the amount of suspending liquid to be reduced whereas the particle volume is kept constant in the foam. The final gas and particle volume fractions are given respectively by: $\phi=\phi_{i}\left(1-\Delta \Omega_{l} / \Omega_{t o t}\right)^{-1}$ and $\varphi_{p}=\varphi_{p_{i}}\left(1-\Delta \Omega_{l} /\left(1-\phi_{i}\right) \Omega_{t o t}\right)^{-1}$. As shown in Fig. 2, the target particle volume fraction can be significantly increased by using this twostep method. With those new parameters, the study of the foam sample can start, as described above ("Study of drainage"). Note that the two-step method induces additional experimental errors in the control of $\phi$ and $\varphi_{p}$.

\section{Parameters:}

In addition to the parameters $D_{b}, d_{p}, \phi$ and $\varphi_{p}$, we will use the so-called confinement parameter, $\lambda$, and the number of particles per foam node, $N$. $\lambda$ compares the particle size to the size of passage through constrictions of the foam network, $d_{c}$ (see Fig. 1), determined in [19] by trapping/release experiments with a single particle in monodisperse foams and by numerical simulations of foam structures,

$$
\lambda=\frac{d_{p}}{d_{c}}=C(\phi) \frac{d_{p}}{D_{b}}
$$

with $C(\phi)=\left(1+0.57(1-\phi)^{0.27}\right) /\left(0.27 \sqrt{(1-\phi)}+3.17(1-\phi)^{2.75}\right) . N$ is obtained by dividing the total number of particles by the total number of foam nodes: $N=\varphi_{p}(1-\phi) D_{b}^{3} / 6 \phi d_{p}^{3}$. By using eq. $1, N$ is obtained as a function of $\lambda$ :

$$
N=\frac{\varphi_{p}(1-\phi)}{6 \phi}\left(\frac{C(\phi)}{\lambda}\right)^{3}
$$




\section{Results and Discussion}

Results for the reduced drainage velocity are presented as a function of $\lambda$ in Fig. 4 . The whole set of data confirms that $\lambda$ is the control parameter for the drainage issue. As $\lambda$ increases, several regimes and transitions are successively observed.

For low and moderate particle volume fractions, i.e. $\varphi_{p} \lesssim 0.40$, one can distinguish five ranges of lambda values: (i) $\lambda \lesssim \lambda^{c}$, where the velocity is almost constant $\left(\varphi_{p} \lesssim 0.16\right)$ or decreases slightly as a function of lambda. (ii) $\lambda^{c} \lesssim \lambda \lesssim \lambda^{*}$, where a significant decrease of the velocity is observed; the decrease is all the more pronounced that the particle volume fraction is low, and for $\varphi_{p} \approx 0.40$ the magnitude of the decrease becomes comparable to that of the preceding regime. (iii) $\lambda \approx \lambda^{*}$, the velocity is minimal. (iv) $\lambda^{*} \lesssim \lambda \lesssim 10$, the drainage velocity increases significantly and reaches values that are close to the velocities in the first regime $\left(\lambda \lesssim \lambda^{c}\right)$. (v) $\lambda \gtrsim 10$, the increase of the velocity is much less pronounced than for the preceding regime.

For the highest investigated particle volume fractions, i.e. $\varphi_{p}>0.40$, the behavior is similar except that the drainage velocity vanishes for moderate lambda values, but takes again a finite value for higher lambda values.

The reported drainage behavior can be interpreted in terms of foam permeability. We introduce the general equation of drainage for aqueous foams:

$$
-\frac{\partial \phi}{\partial t}+\vec{\nabla} \cdot\left(\frac{K_{0}}{\mu_{0}}(\rho \vec{g}-\vec{\nabla} P)\right)=0
$$

where $-\vec{\nabla} P$ is the driving pressure gradient and $K_{0}$ is the foam permeability, which has been shown to depend on the bubble size, gas volume fraction and interfacial mobility $[6,7,23,24]$. For the foams considered in this study the most part of the liquid in contained in the foam nodes, whose characteristic size is $r \approx d_{c} / \alpha$, with $\alpha=2(2 / \sqrt{3}-1)$. It is convenient to introduce the foamaveraged permeability coefficient of a node, $\tilde{k}_{n 0}=K_{0} / r^{2}$. Within the first regime of the free gravity drainage process and assuming an initially uniform gas fraction profile, eq. 3 gives the corresponding constant drainage velocity in the direction of gravity - Darcy velocity $[6,7,23,24]$ :

$$
V_{0}=\frac{K_{0}}{\mu_{0}} \rho g \quad \text { (eq.4) }
$$

Note that this velocity depends on the gas fraction initially set to the foam sample although $\phi(z)$ varies during the linear regime. As already explained, this is due to the fact that the volume of liquid drained out of the foam has flowed through foam areas that have not yet been reached by the 
drainage front, i.e. areas where the gas fraction has remained equal to the initial value $\phi$. For the particle-loaded foams considered in this study, the permeability is expected to depend also on the control parameters $\lambda$ and $\varphi_{p}$ through the partial obstruction of the foam network by trapped particles, i.e. $K \equiv K\left(\phi, D_{b}, \lambda, \varphi_{p}\right)$. Besides, the viscosity of the flowing suspension is expected to depend on $\mu_{0}, \lambda$ and $\varphi_{p}$, i.e. $\mu \equiv \mu\left(\mu_{0}, \lambda, \varphi_{p}\right)$. Finally, the reduced drainage velocity can be expressed as:

$$
\tilde{V}=\frac{V}{V_{0}}=\frac{K}{K_{0}}\left(\frac{\mu}{\mu_{0}}\right)^{-1}
$$

In the following, we estimate $K, \mu$ and $\tilde{V}$, and we discuss all the regimes and transitions described above.

\subsection{Flowing suspension: $\lambda \lesssim \lambda^{c}$}

This regime corresponds to particles suspended in the flowing interstitial liquid. It is expected that the foam permeability is equal to that of the particle-free foam with the same gas fraction, i.e. $K=K_{0}$. Besides, results presented in [18] show that in such conditions the Krieger-Dougherty relationship [25] can be used to estimate the viscosity of the flowing suspension. Consequently, from eq. 5 , the drainage velocity is:

$$
\tilde{V}_{\lambda \leq \lambda^{c}}=\frac{\mu_{0}}{\mu} \simeq\left(1-\frac{\varphi_{p}}{\varphi_{p}^{\text {pack }}(\lambda)}\right)^{2.5 \varphi_{p}^{\text {pack }}(\lambda)}
$$

As already mentioned in [18], in the foam network, confinement effects can be accounted for through a single parameter, $\varphi_{p}^{\text {pack }}(\lambda)$, which represents the particle volume fraction for which the suspension viscosity diverges. This behavior seems to result from the significant interfacial mobility of the confining network, as already observed for the motion of individual particles $[26,27] . \varphi_{p}^{\text {pack }}(\lambda)$ can be significantly reduced with respect to the value for infinite volume (unconfined) conditions, $\varphi_{p}^{\text {pack }}(0)$, and this effect can be estimated by the relation [18]: 


$$
\frac{\varphi_{p}^{\text {pack }}(\lambda)}{\varphi_{p}^{\text {pack }}(0)} \simeq 1-\lambda / 3+0.1 \lambda^{2}
$$

Eq. 6 is plotted in Fig. 9a. Besides, our experimental results indicate that $\lambda^{c} \simeq 0.9$. As it will be explained in the following, for $\lambda>\lambda^{c}$, the evolution of the drainage velocity becomes controlled by the individual capture of particle in the foam network.

\subsection{Maximal frictional drag configuration: $\lambda=\lambda^{*}$}

Now we focus on the minimum value of the drainage velocity, which is observed for $\lambda^{*} \approx 1.75$ and corresponds to the maximal drag force experienced by the draining liquid. It has been already shown that within our experimental conditions, particles capture is complete in the foam samples characterized by $\lambda \gtrsim 1.75$ [21]. This situation has been modeled in considering the flow of the suspending liquid, i.e. with a viscosity $\mu=\mu_{0}$, in the foam network, which is assumed to be not deformed with respect to the particle-free foam, but whose permeability is reduced due to the presence of trapped particle packings that form in the nodes. We introduce the Carman-Kozeny permeability coefficient for packed spheres [28,29], $\tilde{C}_{C K} \approx 10^{-3}$, and we consider the ratio $\varphi_{p} / \varphi_{p}^{\text {pack }}$ as the volume fraction of foam nodes filled with packed particles. In associating the hydraulic contributions of particle-filled and empty volume fractions, we obtain the permeability $K / r^{2} \approx\left[\left(1-\varphi_{p} / \varphi_{p}^{p a c k}\right) / \tilde{k}_{n 0}+\left(\varphi_{p} / \varphi_{p}^{p a c k}\right) /\left(\tilde{C}_{C K} d_{p}^{2} / r^{2}\right)\right]^{-1}$. Therefore, the drainage velocity writes [21]:

$$
\tilde{V}_{N \gg 1}^{*}=\frac{K}{K_{0}} \simeq\left[1-\frac{\varphi_{p}}{\varphi_{p}^{p a c k}}+\frac{\varphi_{p}}{\varphi_{p}^{p a c k}} \frac{\tilde{k}_{n 0}}{\tilde{C}_{C K} \alpha^{2}\left(\lambda^{*}\right)^{2}}\right]^{-1}
$$

Note that for $\lambda=1.75$, the number of particles per node (eq. 2) varies from 1 to 4 as $\varphi_{p}$ varies from 0.1 to 0.4 respectively, so that the packings exist only for intermediate or high particle fractions. In Fig. 5 we report the set of experimental data obtained for $\lambda=\lambda^{*} \approx 1.75$ and we compare it with eq. 8. For the reasons explained above, we restrict this comparison to the highest particle concentrations investigated in this study. Note also that no attempt was done to refine the present model by taking into account the expected effect of $\lambda$ on $\varphi_{p}^{p a c k}$, i.e. we consider that $\varphi_{p}^{\text {pack }} \cong \varphi_{p}^{\text {pack }}(0)$. Although the number of particles per node is rather small, the predicted slope $d \tilde{V}_{N \gg 1}^{*} / d \varphi_{p}$ is in reasonable agreement with experimental data, as shown in Figure 5. 
Obviously, for small particle concentrations, the assumption of particle packings does not hold anymore and another approach should be taken on. For $\varphi_{p} \lesssim 0.1$, there is less than one particle per node and we define the fraction of loaded nodes, $f$, as the number of loaded nodes divided by the total number of foam nodes. As $N<1$ and with the assumption that loaded nodes are filled with only one particle, $f=N$ and the apparent foam permeability can be estimated in considering that, on average, the liquid flows through a fraction $(1-f)$ of unloaded nodes and a fraction $f$ of loaded nodes. This suggests that the liquid does not have preferential paths through unloaded nodes over large distances, and this is conceivable insofar as loaded foam nodes have a moderate drag effect on the liquid, as shown in [30]. By combining in series the contributions of loaded (with a permeability coefficient $\tilde{k}_{n}$ ) and unloaded nodes for the drag experienced by the liquid, we obtain the foam permeability $K / r^{2} \approx\left((1-N) / \tilde{k}_{n 0}+N / \tilde{k}_{n}\right)^{-1}$. In introducing the function $g=\tilde{k}_{n 0} / \tilde{k}_{n}$, the reduced drainage velocity writes:

$$
\tilde{V}_{N \leq 1}^{*} \simeq \frac{K}{K_{0}}=\left[(1-N)+N \cdot g\left(\lambda^{*}\right)\right]^{-1}
$$

In [30] both experiments and numerical simulations have provided a consistent set of data for $g(\lambda)$, and a value equal to 2.5 was reported for $g(\lambda=1.75)$. Equation 9 is plotted in Fig. 5 and shows very good agreement with experimental data for the drainage velocity at small particle concentrations. The evolution for $\tilde{V}^{*}$ in the intermediate concentration range, i.e. $0.1 \lesssim \varphi_{p} \lesssim 0.3$, is more difficult to describe precisely because it involves several configurations with a very small number of particles in foam nodes. In order to describe the experimental data over the full concentration range, we use the following fitting function:

$$
\tilde{V}^{*} \simeq \frac{K}{K_{0}} \simeq 1+(7 / 6) \varphi_{p}^{3 / 2}-2 \varphi_{p}^{1 / 2}
$$

\subsection{Capture transition in the foam network: $\lambda^{c} \lesssim \lambda \lesssim \lambda^{*}$}

Whereas particle capture in the present system is expected to be ideally described by a step function at $\lambda=\lambda^{c}$, the capture and the associated drainage velocity decrease are progressive, from $\lambda=\lambda^{c}$ to $\lambda=\lambda^{*}$ within our experimental conditions, i.e. over a lambda range $\Delta \lambda^{c}=\lambda^{*}-\lambda^{c}$. This behavior is 
partly due to the intrinsic small polydispersity of characteristic sizes in a sample, and partly due to the nature of constrictions that coexist in the foam column, i.e. the constrictions of the bulk foam $\left(d_{c}\right)$ and the constrictions of the foam at wall: $d_{c}^{w} \approx 1.6 d_{c}$. The precise shape of the velocity curve between $\lambda^{c}$ and $\lambda^{*}$ is thus also expected to depend on the proportions of bulk and wall constrictions, i.e. on the column to bubble size ratio. An attempt to take explicitly into account the particle retention curve $R_{p}(\lambda)$ in the modeling of both $K$ and $\mu$ has been made in [21] for $\varphi_{p}=0.16$. Here, new results for the particle retention are provided for $\varphi_{p}=0.40$. As shown in Fig. 6a, the particle retention curve for $\varphi_{p}=0.40$ is similar to the one obtained previously for $\varphi_{p}=0.16$. Therefore, the effect of particle capture on the drainage velocity is expected to be similar for the particle fractions in the range $0-0.40$. Note that the width of the capture transition $\Delta \lambda^{c}$ has a significant effect on the minimum drainage velocity $\tilde{V}^{*}$, as it results from two opposing effects: $\tilde{V}\left(R_{p}<1\right)$ decreases with the proportion $R_{p}$ of captured particles, but in the same time $\tilde{V}\left(R_{p}=1\right)$ increases as $\lambda$ increases (see eq. 8). An example of the global behavior is shown in Fig. 6c.

Insofar as the mechanisms leading to the observed velocity decrease have been already identified in [21], here we adopt of more descriptive approach and we look for a phenomenological fitting function for the velocity decrease between $\lambda=\lambda^{c}$ to $\lambda=\lambda^{*}$. We introduce the normalized parameters: $\hat{\lambda}^{c}=\left(\lambda-\lambda^{c}\right) / \Delta \lambda^{c}$ and $\widehat{V}_{\lambda^{c} \rightarrow \lambda^{*}}=\left(\tilde{V}(\lambda)-\tilde{V}^{*}\right) /\left(\tilde{V}^{c}-\tilde{V}^{*}\right)$, where $\tilde{V}^{c} \equiv \tilde{V}\left(\lambda^{c}\right)$ and $\tilde{V}^{*}$ are given by eqs. 6 and 10 respectively. We plot in Fig. $6 \mathrm{~b}$ the corresponding experimental values for all the investigated particle volume fractions in the range $0-0.40$, and we see that the set of data can be reasonably described by the simple function:

$$
\widehat{V}_{\lambda^{c} \rightarrow \lambda^{*}} \simeq \exp \left(-4 \hat{\lambda}^{c}\right) \quad(\text { eq.11) }
$$

\subsection{Minimal frictional drag configuration: $\lambda \gtrsim 10$}

As small particles, i.e. $\lambda<1$, are incorporated into the foam network, the volume fraction of the interstitial phase is $\phi_{s}=\phi_{\ell}+\phi_{p}$, with $\phi_{\ell}$ (resp. $\phi_{p}$ ) the liquid (resp. particle) volume fraction in foam. As the particle size increases significantly, i.e. $\lambda>2$, the foam network is deformed and particle-induced Plateau borders are created as well as thin films between particle surface and gas [30]. In a certain sense, this geometrical evolution can be considered as the progressive exclusion of the particles from the draining network: the fraction of particle surface area in contact with this network decreases and consequently the drag experienced by the draining liquid reduces 
significantly. This effect is reinforced by the fact that the number of particles is a strong decreasing function of $\lambda$ (see eq. 2). Thus, within the range of particle concentrations investigated in this study, the systems characterized by $\lambda \gg 1$ consist in a small number of excluded particles embedded in a particle-free foam with an effective interstitial volume fraction $\phi_{s}^{e f f} \simeq \phi_{\ell}=\phi_{s}-\phi_{p}$. Therefore, in assuming that the particle-free foam permeability varies as $K_{0} \propto \phi_{\ell}^{3 / 2}$ [31], the velocity in this drainage regime is expected to be:

$$
\tilde{V}_{\lambda \gg 1} \simeq \frac{K_{0}\left(\phi_{s}^{\text {eff }}\right)}{K_{0}\left(\phi_{s}\right)} \simeq\left(1-\varphi_{p}\right)^{3 / 2}
$$

This drainage velocity corresponds to the minimal effect (drag) experienced by the draining liquid when particles are trapped in aqueous foams. Eq. 12 is plotted in Fig. 7 against experimental data obtained for $\lambda>15$, showing very good agreement. In the inset of Fig. 7 is also plotted the ratio $\tilde{V}_{\lambda \gg 1} / \tilde{V}_{\lambda \simeq 0}$ as a function of $\varphi_{p}\left(\widetilde{V}_{\lambda \simeq 0}\right.$ is given by eq. 6 in assuming no confinement effect). It appears that $\tilde{V}_{\lambda \gg 1}$ is the maximum velocity, whatever the particles are flowing or not.

\subsection{Particle Exclusion Transition: $\lambda^{*}<\lambda \lesssim 10$}

Now we consider the transitional regime between the maximal drag configuration $\left(\lambda=\lambda^{*}\right)$ and the minimal drag configuration $(\lambda \gg 1)$. As already explained, the $\lambda=\lambda^{*}$ configuration corresponds to particles that are fully included into the foam network, whereas the $\lambda \gg 1$ configuration consists in particles excluded from the foam network and rather embedded in the bulk foam. Thus, from a geometrical point of view, the transition between those two configurations can be considered as the inclusion/exclusion of the particles with respect to the foam network. Note that within the range $2 \lesssim \lambda \lesssim 10$, the number of particles is divided by more than 100 , and the number of particles per node evolves from $N \approx 1$ to $N \approx 0.01$. Thus, for a given particle concentration, the transitional regime accounts for both the change in particle number and the change in the geometrical configuration of the particles. We define the function $\chi$ that measures the level of viscous drag, i.e. $K^{-1}$ or equivalently $(\tilde{V})^{-1}$, between the two reference configurations: 


$$
\chi=\frac{(\tilde{V})^{-1}-\left(\tilde{V}_{\lambda \gg>1}\right)^{-1}}{\left(\tilde{V}^{*}\right)^{-1}-\left(\tilde{V}_{\lambda \gg 1}\right)^{-1}}
$$

Note that $\chi=0$ as $\tilde{V}=\tilde{V}_{\lambda \gg>1}$ (particles excluded from the network) and $\chi=1$ as $\tilde{V}=\tilde{V}^{*}$ (fully included particles). In eq. 13 , values for $\tilde{V}_{\lambda \gg>1}$ and $\tilde{V}^{*}$ are obtained from eqs 12 and 10 respectively. Calculated values for $\chi$ are plotted in Fig. 8 as a function of $\lambda$, showing a well-defined transition between $\chi=1$ and $\chi=0$ as $\lambda$ increases. As expected, the transitional regime is found to be similar for all the particle volume fractions and it can be described with the same function. Therefore we propose the following phenomenological form to fit the data:

$$
\chi=2.65 \lambda^{-7 / 4} \quad(e q .14)
$$

In combination with eq. 13 , eq. 14 allows for the drainage velocity to be estimated for any particle volume fraction and any $\lambda$ value in the transitional regime. The corresponding values are plotted in Fig. 9a. Further dedicated work would allow understanding the reported transitional regime.

3.6.Jammed state: $\varphi_{p}>\varphi_{p}^{\text {jam }}$

Finally, we address the issue of the vanishing drainage velocity observed for sufficiently high particle concentrations, more precisely when $\varphi_{p}>\varphi_{p}^{j a m}$. For such concentrations, the drainage velocity was not measurable within our experimental conditions, and therefore the foamy suspension appeared to be jammed. In Fig. $9 \mathrm{~b}$ we report the approximate outline of the jammed state, as deduced from our measurements. Note that due to difficulties related to the generation method at high particle concentrations and moderate $\lambda$ values, we were not able to determine precisely all the boundaries. In the following we discuss those boundaries from the theoretical point of view.

For small $\lambda$ values, i.e. $\lambda<1, \varphi_{p}^{\text {jam }}$ identifies to $\varphi_{p}^{\text {pack }}(\lambda)$ given by eq. 7 , which means that the particles collectively jam in the network, i.e. $\mu\left(\varphi_{p}^{\text {pack }}(\lambda)\right) \rightarrow \infty$, and retain the suspending liquid into the porosity of the packing they form. For $\lambda \gtrsim \lambda^{*}$ the situation can be significantly different: the individually trapped particles can induce interface deformations around them, as well as capillary liquid retention even if $\varphi_{p}<\varphi_{p}^{p a c k}$. The study of such complex configurations is clearly beyond the scope of this paper. For the present work, we focus on the particle volume fraction for packing into the foam network in the range of $\lambda \gtrsim \lambda^{*}$. The ideal configuration corresponds to particles that enlarge locally the foam network and fill the network by forming particle chains. Let's call $L_{n n} \approx$ 
$0.36 D_{b}$, the distance between two neighboring node centers $[5,6]$. The maximum number of particles per node that can be put in chains in order to cover the corresponding distance is: $N^{\text {pack }} \simeq 2\left(L_{n n} / d_{p}-1 / 2\right)$. Therefore, using eqs 1 and 2 , the particle volume fraction for packing in chains writes:

$$
\varphi_{p}^{p a c k} \simeq 6 \frac{\phi}{1-\phi}\left(\frac{\lambda}{C(\phi)}\right)^{3}\left(0.72 \frac{C(\phi)}{\lambda}-1\right)
$$

As explained above, we stress that eq. 15 should be considered as an upper bound and that the

jamming of the system could occur for $\varphi_{p}<\varphi_{p}^{p a c k}$. Eq. 15 is plotted in Fig. $9 \mathrm{~b}$ and shows consistency with experimental data. In combination with eqs. 7 and 15 allows understanding the boundaries of the jammed state domain.

\section{Conclusion}

We have tackled the issue of foam drainage in the presence of hydrophilic spherical particles. Through a systematic study of the relevant parameters, i.e. the bubble diameter, the particle diameter, the gas volume fraction and the particle volume fraction, we extent the work performed in previous studies $[18,21,30]$ and we provide a global understanding for the drainage behavior in those complex systems. This will help to interpret drainage data obtained with more specific systems and that cannot be understood from classical drainage laws [23]. In particular, we highlight the effect of both the particle volume fraction and the parameter $\lambda$, that compares the particle size to the size of passage through constrictions in the foam network. For small $\lambda$ values, the suspension drains with an effective viscosity that depends on both $\varphi_{p}$ and $\lambda$ (regime of flowing suspensions). For $\lambda \gtrsim 1$, the particles are captured by constrictions, resulting in a sharp decrease of the velocity (capture transition). Although the particles remain trapped in the foam network as $\lambda$ increases further, the drainage velocity increases for $\lambda \gtrsim 2$, so that a well-defined minimal velocity value is observed for $1<\lambda<2$. This optimal configuration for drainage reduction corresponds to particles that are trapped and fully included in the foam network. As $\lambda$ increases further, the particles become too large to be completely included in the foam network: they become progressively excluded from the network where drainage takes place, so that the drainage velocity increases up to a well-defined asymptotic value which appears to be the maximal drainage velocity. Therefore, the particle 
exclusion transition, which refers to the included / excluded configuration of the particles with respect to the foam network, makes the drainage velocity evolve from its minimal value (fully included particles) to its maximal one (fully excluded particles). This morphological transition remains to be understood in details, but we think that it plays a crucial role in the properties of particulate foamed materials, such as rheology and stability with respect to ripening and coalescence.

We would like to stress that this work could help researchers dealing with foam stabilization via hydrophobic particles to consider properly the effect of the unattached hydrophobic particles in the foam network. Besides, as the paper also reports the conditions for the foam drainage to be blocked, we anticipate that such a result could be applied to formulate rationally new foamed materials. Further work should aim in investigating the drainage of polydisperse granular suspensions that are more likely to be used in practical situations.

\section{Acknowledgements}

We thank D. Hautemayou and C. Mézière for technical support. We gratefully acknowledge financial support from Agence Nationale de la Recherche (Grant no. ANR-13-RMNP-0003-01) and French Space Agency (convention CNES/70980).

\section{References}

[1] R.K. Dhir, M.D. Newlands, A. McCarthy, eds., Use of Foamed Concrete in Construction, Thomas Telford Ltd, London, 2005.

[2] A.R. Studart, U.T. Gonzenbach, E. Tervoort, L.J. Gauckler, J. Am. Ceram. Soc. 89 (2006) 17711789.

[3] A. Wilson, ed., Foams: Physics, Chemistry and Structure, Springer London, London, 1989.

[4] A. Nguyen, H.J. Schulze, Colloidal Science of Flotation, Marcel Dekker, New York, 2003.

[5] D.L. Weaire, S. Hutzler, The Physics of Foams, Oxford University Press, Oxford, 2001.

[6] I. Cantat, S. Cohen-Addad, F. Elias, F. Graner, R. Höhler, O. Pitois, F. Rouyer, Foams: Structure and Dynamics, Oxford University Press, Oxford, 2013.

[7] S. Cohen-Addad, R. Höhler, O. Pitois, Annu. Rev. Fluid Mech. 45 (2013) 241-267.

[8] A.M. Sani, K.K. Mohanty, Colloids Surfaces A Physicochem. Eng. Asp. 340 (2009) 174-181. 
[9] R. Guillermic, A. Salonen, J. Emile, A. Saint-Jalmes, Soft Matter 5 (2009) 4975-4982.

[10] A. Britan, M. Liverts, G. Ben-Dor, S.A. Koehler, N. Bennani, Colloids Surfaces A Physicochem. Eng. Asp. 344 (2009) 15-23.

[11] F. Carn, A. Colin, O. Pitois, M. Vignes-Adler, R. Backov, Langmuir 25 (2009) 7847-7856.

[12] F. Carn, A. Colin, O. Pitois, R. Backov, Soft Matter 8 (2012) 61-65.

[13] I. Lesov, S. Tcholakova, N. Denkov, J. Colloid Interface Sci. 426 (2014) 9-21.

[14] I. Lesov, S. Tcholakova, N. Denkov, RSC Adv. 4 (2014) 811-823.

[15] K. Koczo, L.A. Lobo, D.T. Wasan, J. Colloid Interface Sci. 150 (1992) 492-506.

[16] J. Goyon, F. Bertrand, O. Pitois, G. Ovarlez, Phys. Rev. Lett. 104 (2010) 128301.

[17] A. Salonen, R. Lhermerout, E. Rio, D. Langevin, A. Saint-Jalmes, Soft Matter 8 (2012) 699-706.

[18] B. Haffner, Y. Khidas, O. Pitois, Soft Matter 10 (2014) 3277-3283.

[19] N. Louvet, R. Höhler, O. Pitois, Phys. Rev. E 82 (2010) 041405.

[20] S. Guignot, S. Faure, M. Vignes-Adler, O. Pitois, Chem. Eng. Sci. 65 (2010) 2579-2585.

[21] Y. Khidas, B. Haffner, O. Pitois, Soft Matter 10 (2014) 4137-4141.

[22] D. Weaire, N. Pittet, S. Hutzler, D. Pardal, Phys. Rev. Lett. 71 (1993) 2670-2673.

[23] S.A. Koehler, S. Hilgenfeldt, H.A. Stone, Langmuir 16 (2000) 6327-6341.

[24] E. Lorenceau, N. Louvet, F. Rouyer, O. Pitois, Eur. Phys. J. E 28 (2009) 293-304.

[25] I.M. Krieger, T.J. Dougherty, J. Rheol. (N. Y. N. Y). 3 (1959) 137-154.

[26] O. Pitois, C. Fritz, L. Pasol, M. Vignes-Adler, Phys. Fluids 21 (2009) 103304.

[27] F. Rouyer, C. Fritz, O. Pitois, Soft Matter 6 (2010) 3863-3869.

[28] J. Kozeny, Sitzungsber Akad. Wiss. 136 (1927) 271-306.

[29] P.C. Carman, Trans. Instn Chem. Engrs 15 (1937) 150-166.

[30] F. Rouyer, B. Haffner, N. Louvet, Y. Khidas, O. Pitois, Soft Matter 10 (2014) 6990-6998.

[31] S.A. Koehler, S. Hilgenfeldt, H.A. Stone, Phys. Rev. Lett. 82 (1999) 4232-4235. 


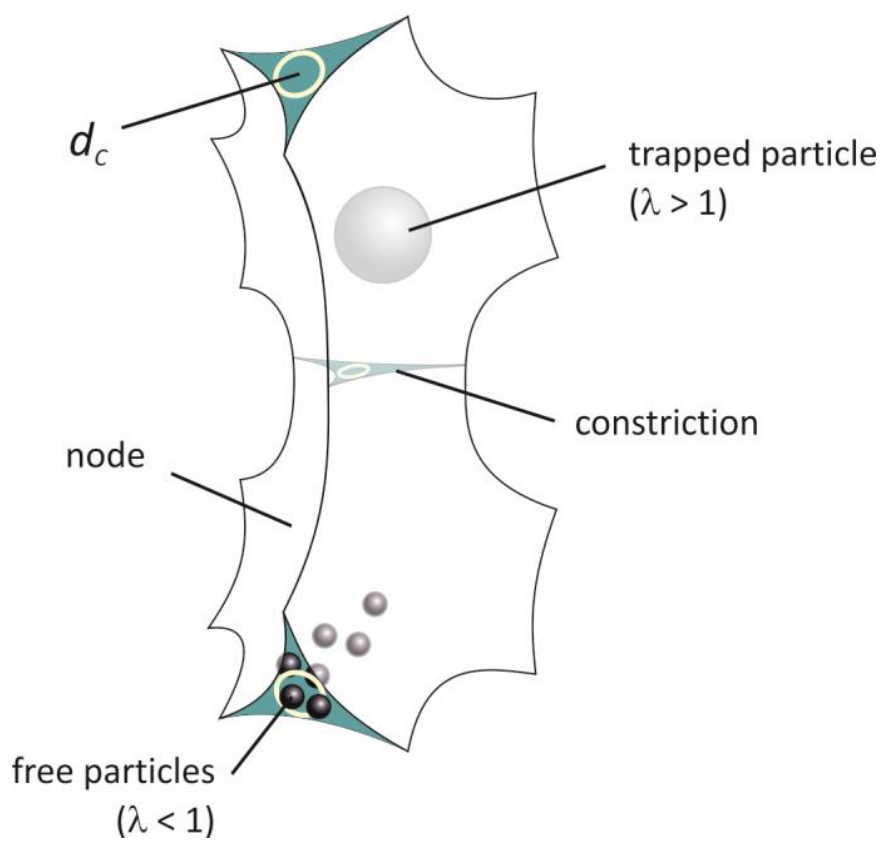

Figure 1: The interstitial network of aqueous foams consists in nodes connected by constrictions. Particles suspended in the interstitial fluid can be either freely transported through the constrictions or trapped by constrictions. This behavior is described using the so-called confinement parameter, $\lambda$, that compares the particle size to the size of passage through those constrictions, $d_{c}$. 


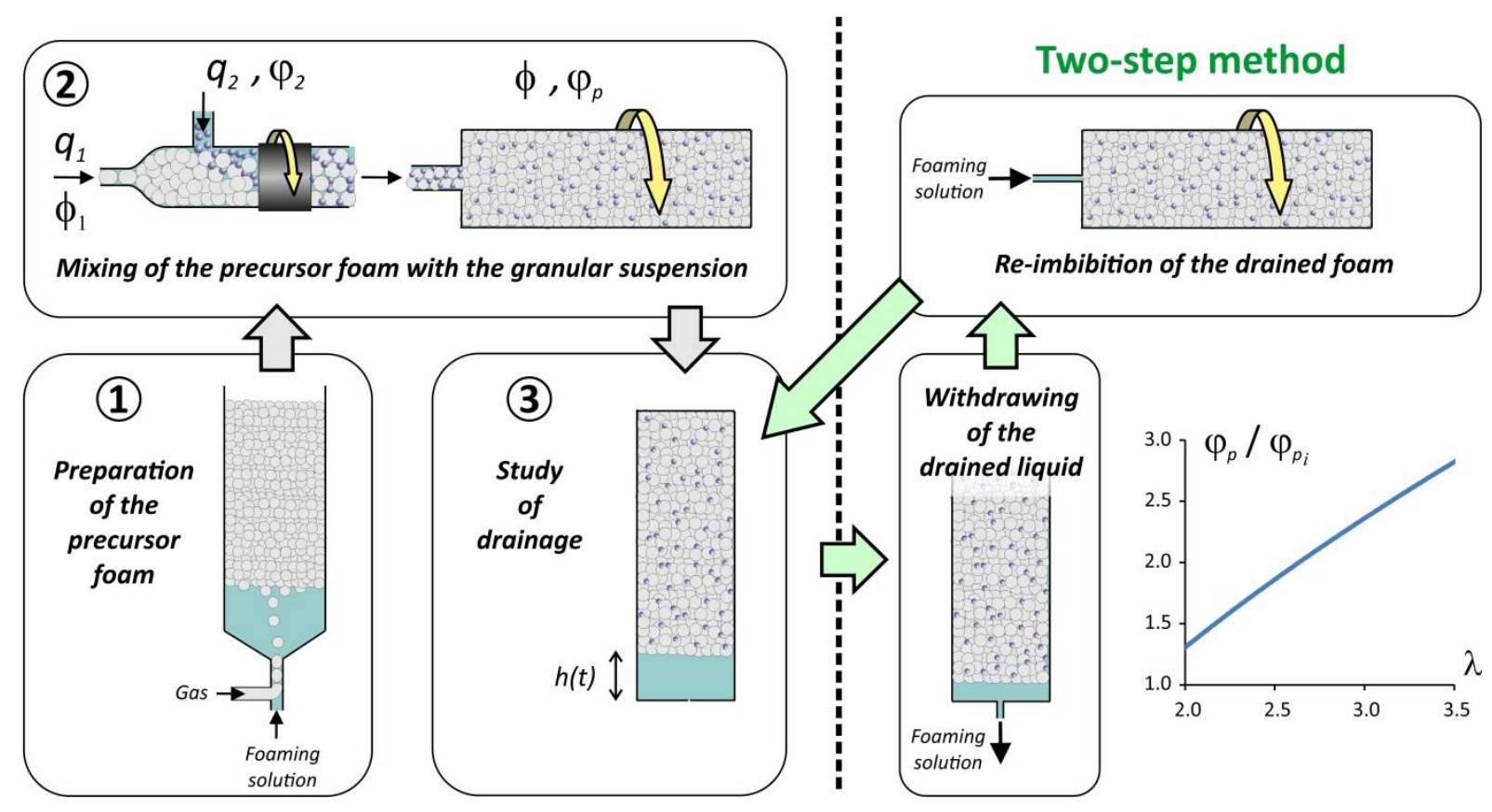

Figure 2: Generation method for foamy suspensions. Precursor foam is produced in a column (1) before being co-injected with the particle suspension through the mixing device (2). The tuning of flow rates and volume fractions of both foam and suspension allows for the foamy suspension to be controlled during the mixing stage. The sample is continuously introduced and stored in a rotating horizontal column in order to avoid drainage during the preparation. Then the column is turned to the vertical and the study of drainage starts (3). The "two-step" method can be used for samples with high particle concentrations and large particles that are trapped in the foam network. In those cases, the liquid drained after (3) is first withdrawn from the column. Then the horizontal rotation motion is restored and a given volume of liquid is re-injected in the rotating column in order to adjust the final particle volume fraction. After that second step, the study of drainage starts (3). The graph shows the maximal increase of the particle volume fraction (with respect to the one-step method) resulting from the use of the two-step method as a function of the target lambda value. 


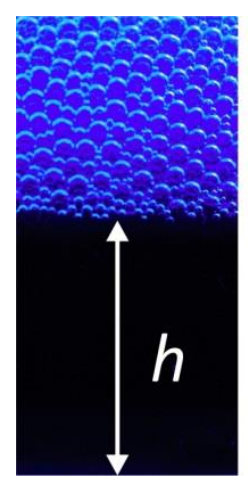

(a)

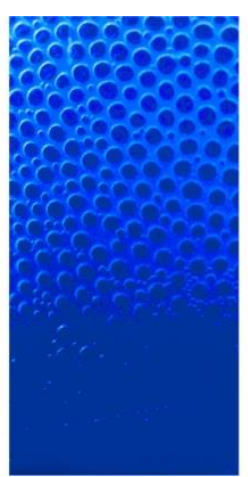

(b)

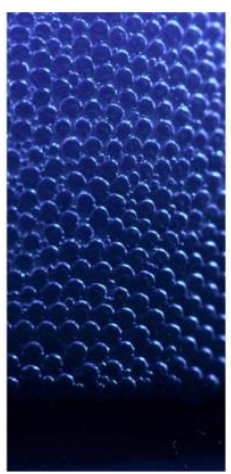

(c)

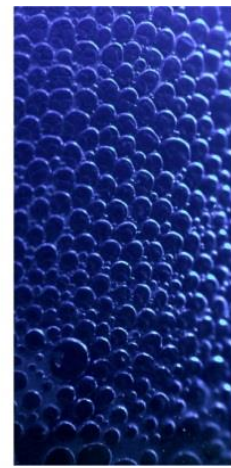

(d)

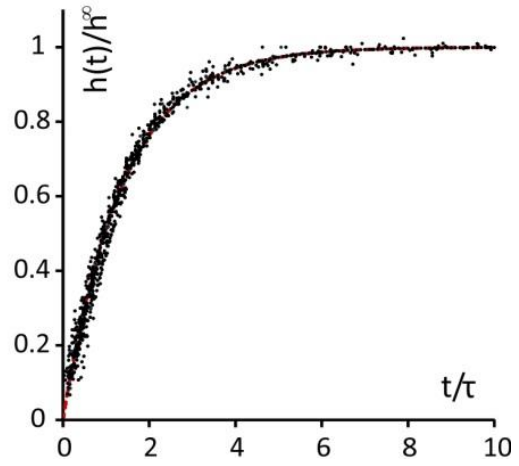

(e)

Figure 3: Study of foam drainage. (a) to (d) Close-up at the bottom of foam samples with bubble size $D_{b} \simeq 660 \mu \mathrm{m}$ and $\phi=0.9$, after a drainage time equal to $500 \mathrm{~s}$ : (a) No particle, $\varphi_{p}=0$; (b) Particles flow out of the foam, $\varphi_{p} \simeq 0.40$ and $d_{p} \simeq 20 \mu \mathrm{m}$; (c) Particles are trapped in the foam, $\varphi_{p} \simeq 0.35$ and $d_{p} \simeq 80 \mu \mathrm{m}$; (d) Jammed foam, $\varphi_{p} \simeq 0.49$ and $d_{p} \simeq 40 \mu \mathrm{m}$.

(e) Reduced height of liquid/suspension drained at the bottom of the foam column as a function of the reduced time, for more than 60 foam samples. $h^{\infty}$ is the drained height measured at long time and $\tau$ the time corresponding to $h(\tau) / h^{\infty}=1 / 2$. 


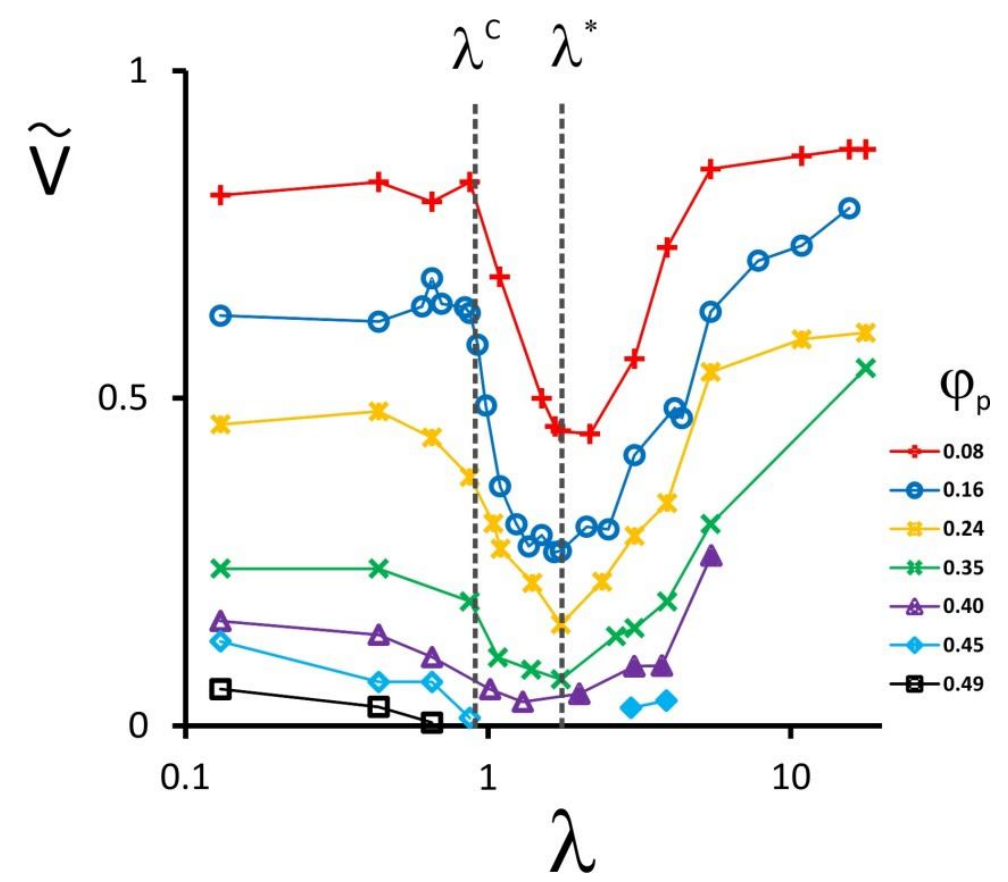

Figure 4: Reduced drainage velocity of foamy suspensions as a function of the confinement parameter, for several particle volume fractions. Full symbols indicate measurements for which the "two-step" method has been used. The two particular values $\lambda^{c}$ and $\lambda^{*}$ refer respectively to the end of the flowing suspension regime and to the minimal drainage velocity. 


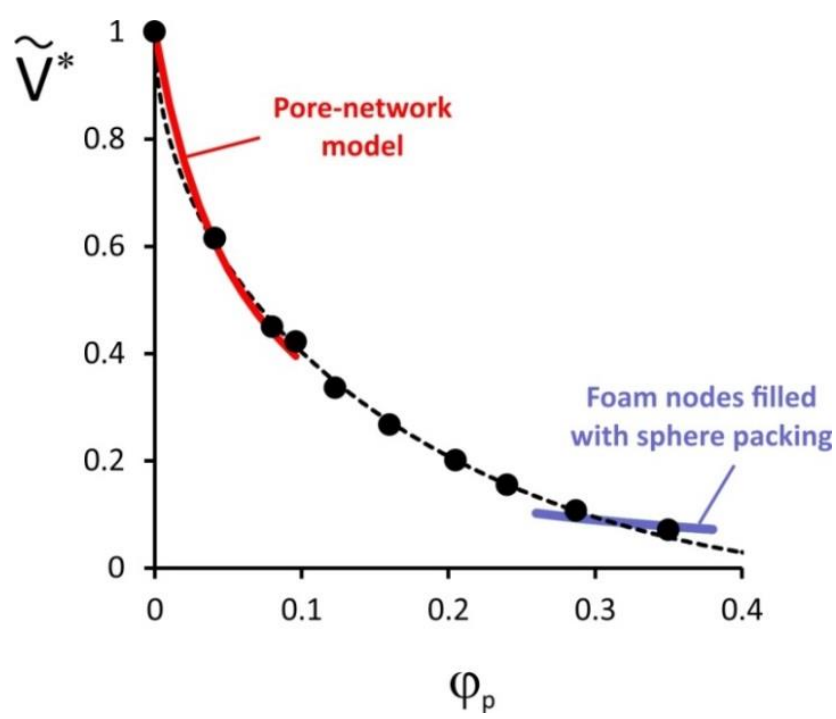

Figure 5: Minimum drainage velocity $\left(\lambda \approx \lambda^{*}\right.$ in Figure 4$)$ as a function of particle volume fraction. The blue line corresponds to eq. 8 plotted with $\varphi_{p}^{\text {pack }}=0.6, \tilde{C}_{C K}=0.001, \alpha=0.31, \lambda^{*}=1.75$, $\tilde{k}_{n}=1 / 200$. The red line corresponds to eq. 9 plotted with $g\left(\lambda^{*}\right) \simeq 2.5$. The dotted line corresponds to eq. 10. 


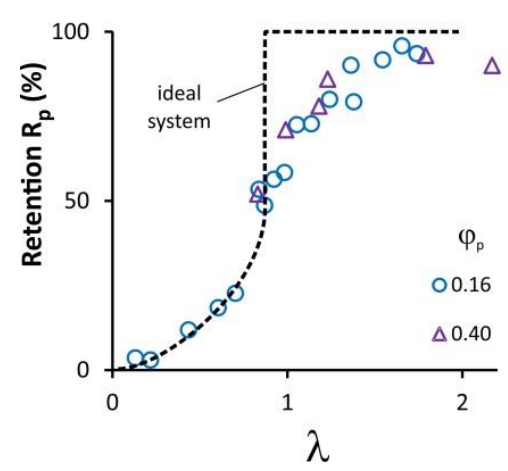

(a)

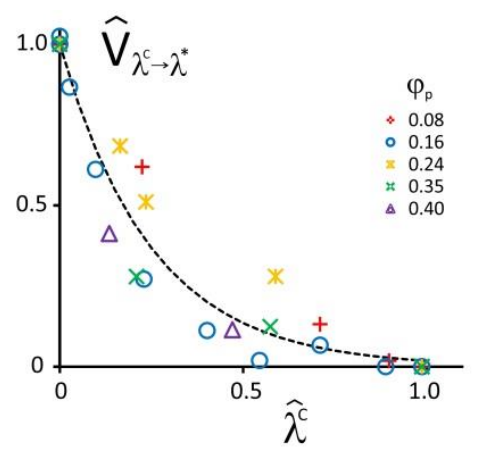

(b)

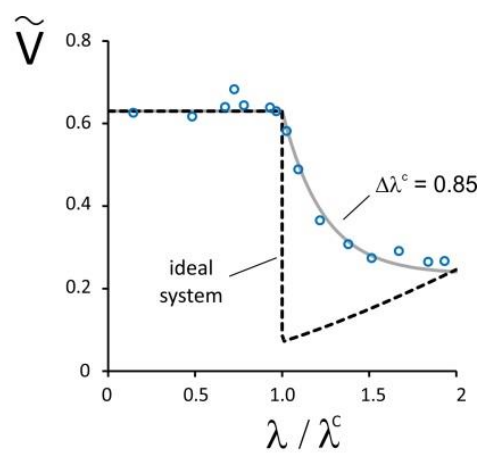

(c)

Figure 6: (a) Proportion of particles retained in the foam after drainage as a function of the confinement parameter, for two particle volume fractions (0.16 and 0.40). The dotted line correspond to the retention curve expected for an ideal system, i.e. $\Delta \lambda^{c}=0$.

(b) Normalized velocities as a function of the normalized confinement parameter for the transition induced by particle capture in foam samples. The dotted line corresponds to eq. 11.

(c) Example of the effect of the capture transition on the minimal drainage velocity $\left(\varphi_{p}=0.16\right) . \widetilde{V}_{\lambda \leq \lambda^{c}}$ is given by eq. 6 (with $\varphi_{p}^{\text {pack }} \cong 0.6$ ), values for $V\left(\lambda \geq \lambda^{*}\right.$ ) are given by eq. 8 with $\tilde{k}_{n}=1 / 300$.

Transition curves are plotted using eq. 11 in considering two values for $\Delta \lambda^{c}: 0$ (ideal system) and 0.85 . 


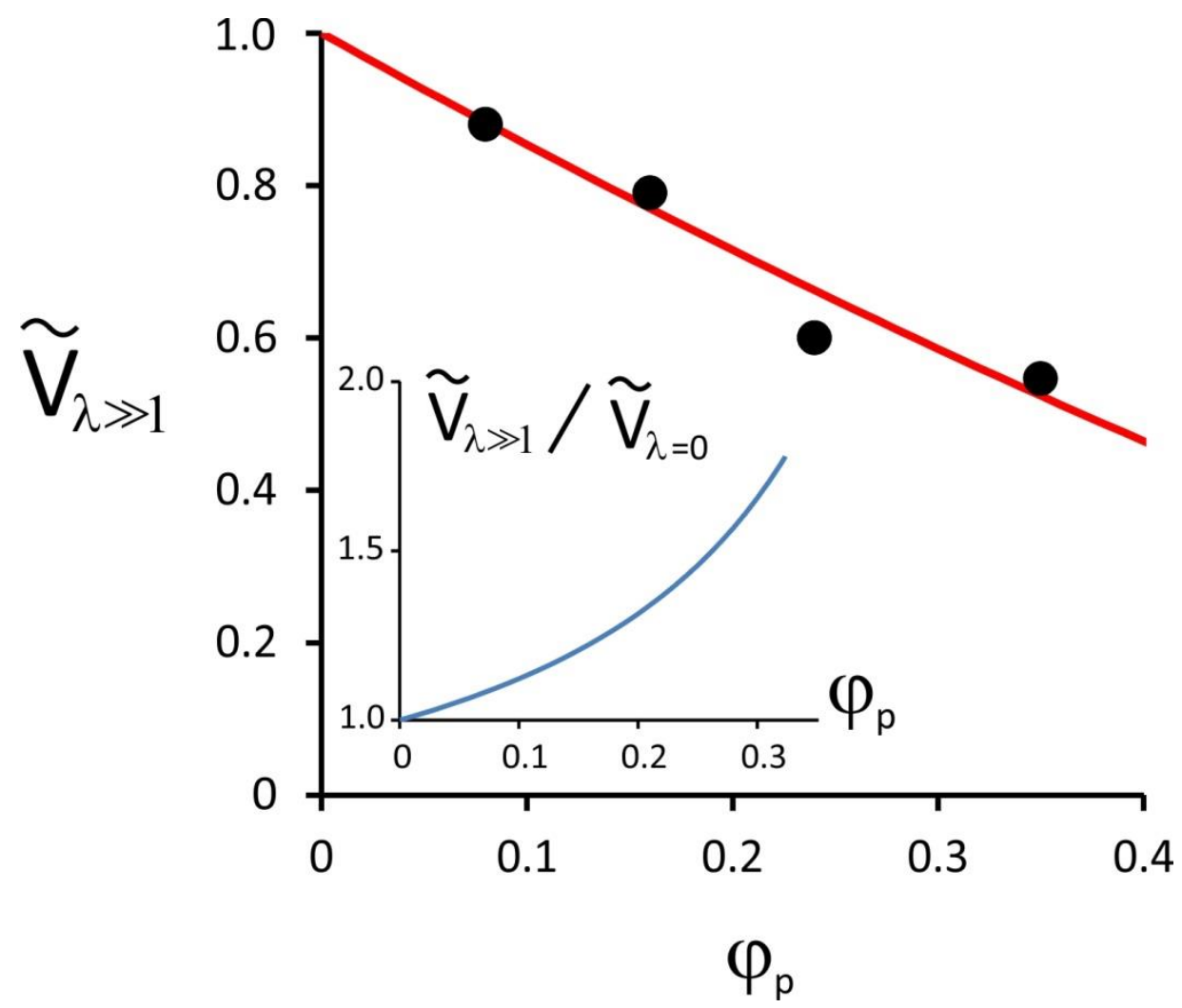

Figure 7: Reduced drainage velocity as a function of particle volume fraction for samples characterized by a large value of the confinement parameter $(\lambda>15)$. The red line corresponds to eq. 12. Inset: the ratio of drainage velocities given by eq. 12 and eq. 6 (assuming no confinement effect, i.e. $\varphi_{p}^{\text {pack }} \cong 0.6$ ) as a function of the particle volume fraction. 


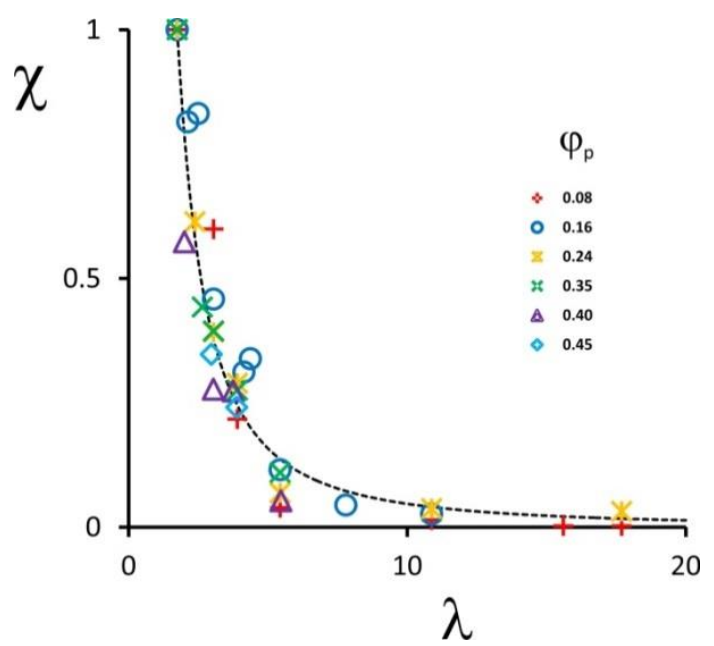

Figure 8: Normalized transition function between the maximal drag configuration ( $\lambda=\lambda^{*}$ and particles are fully included in the foam network), and the minimal drag configuration ( $\lambda \gg 1$ and the particles are excluded from the network). The symbols correspond to values obtained from experimental data and eq. 13. The dotted line corresponds to eq. 14. 


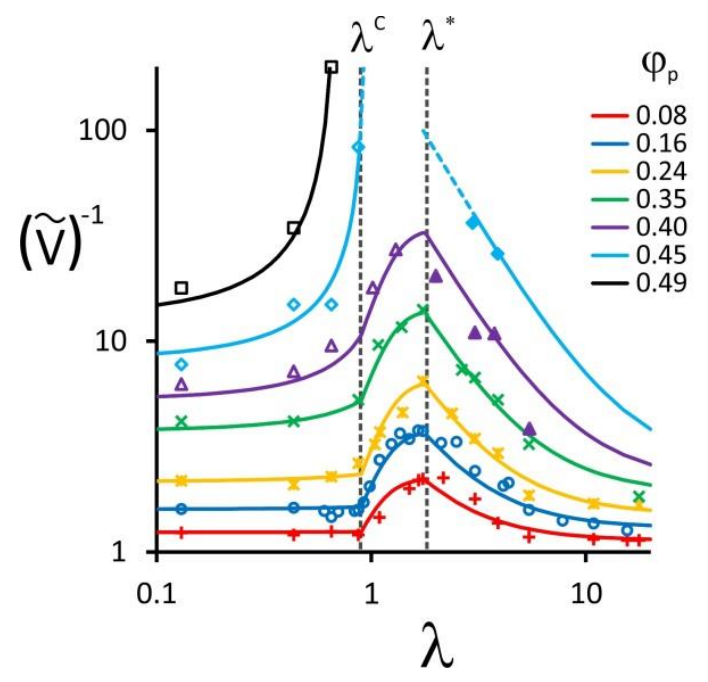

(a)

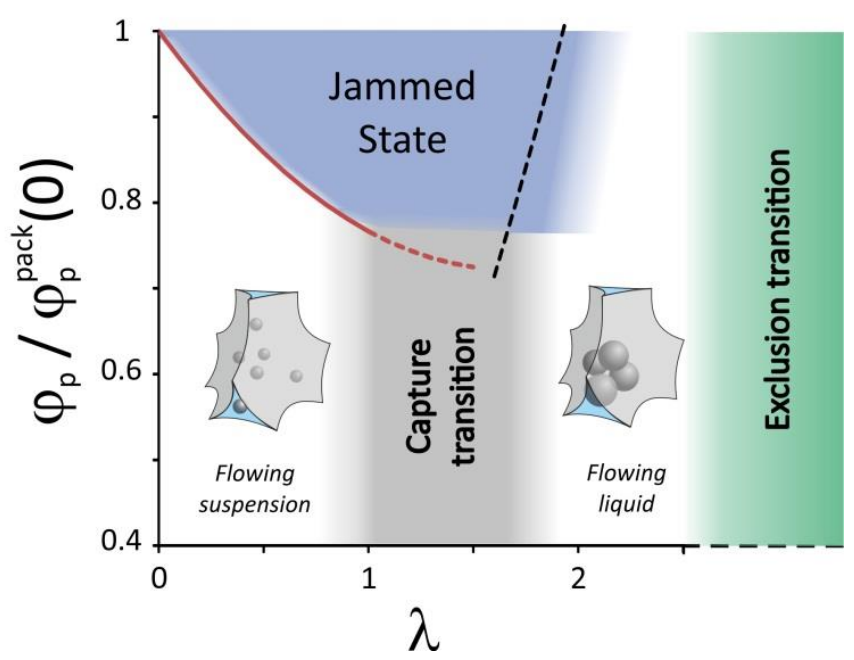

(b)

Figure 9: (a) Reduced viscous drag (inverse of the reduced drainage velocity) of foamy suspensions as a function of the confinement parameter, for several particle volume fractions. The two particular values $\lambda^{c}$ and $\lambda^{*}$ refer respectively to the end of the flowing suspension regime and to the minimal drainage velocity (maximal drag). The lines correspond to eq. 6 for $\lambda \leq \lambda^{c}$, to eq. 11 for $\lambda^{c} \leq \lambda \leq \lambda^{*}$ and to eq. 12 for $\lambda \geq \lambda^{*}$.

(b) Diagram of the reduced particle volume fraction $\left(\varphi_{p}^{\text {pack }}(0)\right.$ is the packing volume fraction without confinement effect) as a function of the confinement parameter in the foam network. It shows the jammed state domain deduced from experiments. The red line corresponds to eq. 7 and the black dotted line corresponds to eq. 15. 